\title{
The microlensing probability of the pulsars located in globular clusters
}

\author{
Tanya Larchenkova \\ Astro Space Center of P.N.Lebedev Physical Institute, Profsoyuznaya \\ 84/32, Moscow 117810 RUSSIA
}

\begin{abstract}
We have used the effect of Shapiro delay of the pulsar radiation in the gravitational lensing approximation. The microlensing probabilities for pulsars located in globular clusters 47 Tuc (NGC 104) and M15 (7078) in the case of rather large impact parameters was calculated.
\end{abstract}

The problem of the dark matter detection in our Galaxy is very important nowadays. General relativistic effects - gravitational lensing and time delay of pulsar's signal in the gravitational field of a point mass can help, in principle, to solve this problem. The gravitational lensing manifestation is the magnification of the radiation flux which will be accompanied the time delay of the pulsar's signal (Krauss \& Small 1991). For instance, unusual shape of residuals of the pulsar B0525+21 may be explained in terms of such a relativistic time delay in gravitational field of a massive compact object (Larchenkova \& Doroshenko 1995).

The time delay in propagation of pulsar's pulse consists of two terms: geometrical time delay, and Shapiro delay (Kopeikin et al. 1999). In the case of pulsar timing observations the geometrical time delay can be neglected. To calculate the Shapiro delay we use the gravitational lensing approximation (Larchenkova $\&$ Doroshenko 1995). Since the pulsar and lens are moving the Shapiro delay is a function of the observation time. The delay reaches a maximum value at the instant corresponding to the minimal value of the impact parameter $d$. For example, assuming $d=100 \mathrm{AU}$, the relative velocity $V_{P}=100 \mathrm{~km} / \mathrm{s}$ of the pulsar projected in the plane of the sky, with respect to a single gravitating mass $M=0.1 M_{\odot}$, and precision of the timing observation $0.5 \mu s$, the necessary observation time of the effect is around 8 years.

Eight pulsars and twenty millisecond pulsars have been observed in globular cluster M15 and 47 Tuc. Almost all pulsars are located in the core of the clusters. In the present paper we are interested in estimation of microlensing probability of pulsars located in a globular cluster. Two cases are possible - a lens in the globular cluster, and one located outside it somewhere in the Galaxy. We consider a globular cluster as a self-gravitating isothermal sphere made of identical stars and use $\frac{1}{1+r^{2}}$ model of mass density, where $r$ is the distance to the cluster's center w.r.t. the core's radius $r_{c}$. Note that the probability, $P$, should be a function of the total mass of lensing objects and can not depend on the individual lens mass. If the pulsar is located at the center of the cluster then one can estimate the ratio $P / f^{2}$ being equal to $4.6 \cdot 10^{-8}$ for 47 Tuc and $3.7 \cdot 10^{-8}$ for M15. $R_{E}$ is the Einstein radius. 
We assume the mass function to be independent of the position and all lensing objects have the same mass $0.1 M_{\odot}$. This assumption may be applied because the mass fraction of low-mass white dwarfs is $\sim 50 \%$ in 47 Tuc (Heggie 1995); the white dwarfs constitute most (about 85\%) of the mass of M15 (Gebhardt 1997).

If we know the lens mass, $M$, the impact parameter, $d$, and the system geometry then one can estimate parameter $f$. Hence, we can derive $P$. For $M=0.1 M_{\odot}$ and $d=100 \mathrm{AU}$ parameter $f$ is around 1000 .

To estimate the lensing probability, $P$, on objects of Milky Way we use an empirical model of Bahcall \& Soniera (1980) for the density of source in the plane of the Galaxy and the model of the isotermal sphere with a core for Galactic halo. The ratio $P / f^{2}$ have been derived $1.5 \cdot 10^{-7}$ and $5.5 \cdot 10^{-7}$ for the pulsar in 47 Tuc and in M15, correspondingly. Assuming all lenses have the same mass also being equal to $0.1 M_{\odot}$, as the Galactic halo, probably, consists of red dwarfs or even, brown dwarfs, we can estimate the numerical value of parameter $f$ and therefore the probability.

During 8 years of the observations the number of expected lensing events for parameters $M=0.1 M_{\odot}, d=100 A U$ and the different location of lens is given below.

\begin{tabular}{|c|c|c|c|c|}
\hline & \multicolumn{2}{|c|}{ Source in center of M15 } & \multicolumn{3}{c|}{ Source in center of 47 Tuc } \\
\hline & lens in M15 & lens in MW & lens in 47 Tuc & lens in MW \\
\hline Events & 0.2 & 3 & 0.6 & 2 \\
\hline
\end{tabular}

Several pulsars exhibit the presence of some unexplained timing noise. Weak gravitational lensing by galactic objects may explain some symmetrical distortions of the timing residuals. From our calculation it follows that one expect less number of lensing events by objects located in a globular cluster than by objects being outside it. This result can be explaned more high value of the probability and also more high numerical value of the dispersion velocity in the halo.

The objective observational difficulties of rather weak Shapiro effect at the large time scale deal with the timing noise. But we believe that in near future the accuracy $\sim 100$ ns will be attain even for pulsars with weak flux. The power spectrum of Shapiro effect will allow to discern it from low frequency timing noise.

\section{References}

Bahcall, J. N., \& Soneira, R. M. 1980, ApJS, 44, 73

Gebhardt, K., Pryor, C., Williams, T. B., Hesser, J. E., \& Stetson, P. B. 1997, AJ, 113,1026

Heggie, D. C., \& Hut, P. 1996, in IAU Symp. 174, Dark Matter in Globular Clusters, 303

Kopeikin, S.M., Schafer,G., Gwinn, C.R., Eubanks, T.M. 1999, Phys.Rev.D, 59

Krauss, L. M., \& Small, T. A. 1991, ApJ, 378, 22

Larchenkova, T. I., \& Doroshenko, O. V. 1995, A\&A, 297, 607 